\title{
Efficient Knoevenagel Condensation Reactions Catalyzed by Activated Gel Zirconium(IV) Oxide
}

\author{
Mohammad Reza Poor Heravi and S. Piri \\ Department of Chemistry, Payame Noor University, Tehran 19395-4697, Iran \\ Correspondence should be addressed to Mohammad Reza Poor Heravi; heravimr@yahoo.com
}

Received 1 February 2012; Revised 15 June 2012; Accepted 23 June 2012

Academic Editor: Albert Demonceau

Copyright (C) 2013 M. R. Poor Heravi and S. Piri. This is an open access article distributed under the Creative Commons Attribution License, which permits unrestricted use, distribution, and reproduction in any medium, provided the original work is properly cited.

The condensation reaction has been carried out very conveniently to obtain the corresponding substituted $2,2^{\prime}$ (arylmethylene)bis(3-hydroxy-5,5-dimethylcyclohex-2-enone) in excellent yields. The reaction conditions are very mild and applicable to various aldehydes as well as active methylene compounds.

\section{Introduction}

In past decades, several carbon-carbon bond-forming reactions have been discovered, and their applications in organic chemistry have also been well documented in the literature [1-6]. Base-catalyzed transformations are frequently used both on small scale as well as large scale in organic synthesis, for example, in Aldol [7], Knoevenagel [8], Henry [9], and Michael [10] reactions. To catalyze these processes, organic amines, alkali alkoxides, and alkali hydroxides are commonly used as a homogeneous phase with the reagents. Although effective, these reagents are difficult to separate and, in many cases, are not recycled. To alleviate this problem, solid basic catalysts have been developed utilizing either inorganic solid materials, such as base metal oxides and carbonates, or by supporting organic bases, for example, amines, on inorganic or polymeric supports. This approach has attracted intense interest and has been reviewed extensively $[11,12]$. Effective heterogeneous base catalysis have been found for Aldol [1315], Knoevenagel [16-18], Henry [19, 20], and Michael [21] reactions, and, in many, cases the solid base is recyclable [22]. Chalcones are the main precursors for the biosynthesis of flavonoids, which are frequent components of the human diet [23]. Recently, studies on biological evaluation of chalcones revealed some to be anticancer $[24,25]$, anti-inflammatory $[26,27]$, antimitotic [28], antitubercular [29], cardiovascular [30], cell differentiation inducing [31], nitric oxide regulation modulatory [32], and antihyperglycemic agents [33]. Of the many methods available for the synthesis of chalcones, the most widely used is the base-catalyzed Claisen-Schmidt reaction in which the condensation of a ketone with an aldehyde is carried out in the presence of aq. $\mathrm{NaOH}$ [34], $\mathrm{KOH}$ [35], $\mathrm{Ba}(\mathrm{OH})_{2}$ [36, 37], hydrotalcites [38], LiHDMS [39], and calcined $\mathrm{NaNO}_{3}$ /natural phosphates [40]. The acidcatalyzed methodologies include the use of $\mathrm{AlCl}_{3}$ [40], dry $\mathrm{HCl}$ [41], $\mathrm{Zn}$ (bpy) $(\mathrm{OAC})_{2}$ [42], $\mathrm{TiCl}_{4}$ [43], $\mathrm{CpZrH}_{2} / \mathrm{NiCl}_{2}$ [44], Zeolites [45], $\mathrm{RuCl}_{3}$ [46], and Selectfluor [47].

\section{Experimental}

Aldehydes were distilled before use. Melting points were determined using a Linkman HF591 heating stage, used in conjunction with a TC92 controller, and reuncorrected. NMR spectra were recorded using a Bruker DRX500 machine at room temperature. ${ }^{1} \mathrm{H}$ and ${ }^{13} \mathrm{C}$ NMR spectra were measured using deuterochloroform as solvent, and chemical shifts were measured relatives to residual solvent as an internal standard and are expressed in parts per million $(\delta)$. Mass spectra were obtained using a Micro Mass LCT machine in ES or EI mode. Infrared spectra were measured on a Perkin Elmer Paragon 100 FT-IR spectrometer.

2.1. Typical Procedure for $2,2^{\prime}$-(Phenylmethylene)bis(3-hydroxy-5,5-dimethylcyclohex-2-enone) (3a). A mixture of benzaldehyde (1) (1 mmol) dimedone (2) $(1 \mathrm{mmol}), \mathrm{ZrOCl}_{2}$ THF $(0.048 \mathrm{~g}, 0.15 \mathrm{mmol})$, and sodium amide $(0.005 \mathrm{~g}$, 
$0.15 \mathrm{mmol}$ ) was added at room temperature. The solution was stirred for $20 \mathrm{~min}$ at room temperature. After dilution with moist ether $(10 \mathrm{~mL})$, the solution was washed with water $(3 \times$ $20 \mathrm{~mL}$ ) to discharge the colour and the $\mathrm{ZrOCl}_{2}$ and sodium amide. The ethereal solution obtained after extraction was dried over anhydrous $\mathrm{Na}_{2} \mathrm{SO}_{4}$ and evaporated under reduced pressure. The crude mixture was passed through a silica gel column chromatography to afford desired product (3a).

2.2. 2,2' -(Phenylmethylene)bis(3-hydroxy-5,5-dimethylcyclohex-2-enone) (3a). FT-IR (KBr) $v_{\max } 3435,3093,2982,1721$ $(\mathrm{C}=\mathrm{O}), 1558,1441,1366,1237,919,855$ and $821 \mathrm{~cm}^{-1} ;{ }^{1} \mathrm{H}$ $\mathrm{NMR}\left(500 \mathrm{MHz}, \mathrm{CDCl}_{3}\right): \delta 1.10(\mathrm{~s}, 6 \mathrm{H}), 1.24(\mathrm{~s}, 6 \mathrm{H}), 2.38$ $(\mathrm{m}, 8 \mathrm{H}), 5.54(\mathrm{~s}, 1 \mathrm{H}), 7.29$ (ddd, $\mathrm{J}=8.5,2.5$ and $1.5 \mathrm{~Hz}, 1 \mathrm{H})$, $7.32(\mathrm{~m}, \mathrm{~J}=8.5 \mathrm{~Hz}, 2 \mathrm{H})$ and $7.36(\mathrm{dd}, \mathrm{J}=8.5$ and $1.5 \mathrm{~Hz}, 2 \mathrm{H})$, 11.89 (s, ex. with $\left.\mathrm{D}_{2} \mathrm{O}, 2 \mathrm{H}\right) ;{ }^{13} \mathrm{C}$ NMR $\left(126 \mathrm{MHz} \mathrm{CDCl}_{3}\right): \delta$ 27.4, 29.64, 31.40, 32.72, 46.43, 47.40, 115.57, 125.83, 126.75, 128.20, 138.2, $196.42(\mathrm{C}=\mathrm{O}), 190.13(\mathrm{C}=\mathrm{O})$; (EI) Found: $\mathrm{M}^{+}$, 368.2005, $\mathrm{C}_{23} \mathrm{H}_{28} \mathrm{O}_{4}$ requires $\mathrm{M}^{+}, 368.3202$; LRMS m/z (EI): $368\left(\mathrm{M}^{+}, 93 \%\right), 293\left(\mathrm{M}-\mathrm{C}_{6} \mathrm{H}_{5}, 45 \%\right)$. Elemental analysis: found (\%): C, 75.12; $\mathrm{H}, 7.86$. Calcd. for $\mathrm{C}_{23} \mathrm{H}_{28} \mathrm{O}_{4}$ : C, 74.97; $\mathrm{H}, 7.66$.

2.3. 2, 2'-((4-Chlorophenyl)methylene)bis(3-hydroxy-5,5-dimethylcyclohex-2-enone) (3b). FT-IR (KBr) $v_{\max } 3456,3100$, 2978, $1719(\mathrm{C}=\mathrm{O}), 1550,1453,1355,1264,918,850$ and $825 \mathrm{~cm}^{-1} ;{ }^{1} \mathrm{H}$ NMR $\left(500 \mathrm{MHz}, \mathrm{CDCl}_{3}\right): \delta 0.85(\mathrm{~s}, 6 \mathrm{H}), 1.22$ (s, 6H), $2.40(\mathrm{~m}, 8 \mathrm{H}), 5.55(\mathrm{~s}, 1 \mathrm{H}), 7.02(\mathrm{~d}, \mathrm{~J}=8.03 \mathrm{~Hz}, 1 \mathrm{H})$, $7.24(\mathrm{~m}, \mathrm{~J}=8.03 \mathrm{~Hz}, 2 \mathrm{H})$ and $11.87\left(\mathrm{~s}\right.$, ex. with $\left.\mathrm{D}_{2} \mathrm{O}, 2 \mathrm{H}\right) ;{ }^{13} \mathrm{C}$ NMR (126 MHz, $\mathrm{CDCl}_{3}$ ): $\delta$ 27.39, 29.59, 31.40, 32.38, 46.39, 47.01, 115.31, 128.18, 131.55, 136.68, $189.42(\mathrm{C}=\mathrm{O}), 190.63$ $(\mathrm{C}=\mathrm{O})$; (EI) Found: $\mathrm{M}^{+}, 402.3805, \mathrm{C}_{23} \mathrm{H}_{27} \mathrm{ClO}_{4}$ requires $\mathrm{M}^{+}$, 402.1678; LRMS m/z (EI): $402\left(\mathrm{M}^{+}, 86 \%\right), 325\left(\mathrm{M}-\mathrm{C}_{6} \mathrm{H}_{5}\right.$, 45\%). Elemental analysis: found (\%): C, 68.88; H, 6.86; Cl, 8.91. Calcd. for $\mathrm{C}_{23} \mathrm{H}_{27} \mathrm{ClO}_{4}$ : C, 68.56; $\mathrm{H}, 6.75 ; \mathrm{Cl}, 8.80$.

2.4. 2, $2^{\prime}$-((3-Nitrophenyl)methylene)bis(3-hydroxy-5,5-dimethylcyclohex-2-enone) (3c). FT-IR (KBr) $v_{\max } 3356,3103$, 2965, 1704 (C=O), 1555, 1450, 1345, 1254, 967, 835 and $811 \mathrm{~cm}^{-1} ;{ }^{1} \mathrm{H}$ NMR $\left(500 \mathrm{MHz}, \mathrm{CDCl}_{3}\right): \delta 1.10(\mathrm{~s}, 6 \mathrm{H})$, $1.41(\mathrm{~s}, 6 \mathrm{H}), 2.38(\mathrm{~m}, 8 \mathrm{H}), 4.66(\mathrm{~s}, 1 \mathrm{H}), 7.41-7.56(\mathrm{~m}$, $4 \mathrm{H})$ and $12.01\left(\mathrm{~s}\right.$, ex. with $\left.\mathrm{D}_{2} \mathrm{O}, 2 \mathrm{H}\right) ;{ }^{13} \mathrm{C}$ NMR $(126 \mathrm{MHz}$, $\left.\mathrm{CDCl}_{3}\right): \delta 27.32,29.67,31.41,32.90,47.01,128.99,132.93$, 144.04, 151.32, $184.47(\mathrm{C}=\mathrm{O}), 190.88(\mathrm{C}=\mathrm{O})$; (EI) Found: $\mathrm{M}^{+}, 413.1805, \mathrm{C}_{23} \mathrm{H}_{27} \mathrm{NO}_{6}$ requires $\mathrm{M}^{+}, 413.1618$; LRMS $\mathrm{m} / \mathrm{z}$ (EI): $413\left(\mathrm{M}^{+}, 56 \%\right), 336\left(\mathrm{M}-\mathrm{C}_{6} \mathrm{H}_{5}, 45 \%\right)$. Elemental analysis: found (\%): C, 66.91; H, 6.86; N, 3.41. Calcd. for $\mathrm{C}_{23} \mathrm{H}_{27} \mathrm{NO}_{6}$ : C, 66.81; H, 6.58; N, 3.39.

2.5. 2, 2'-((4-Nitrophenyl)methylene)bis(3-hydroxy-5,5-dimethylcyclohex-2-enone) (3d). FT-IR (KBr) $v_{\max } 3397,3096$, 2923, $1705(\mathrm{C}=\mathrm{O}), 1551,1455,1355,1250,927,825$ and $819 \mathrm{~cm}^{-1} ;{ }^{1} \mathrm{H} \mathrm{NMR}\left(500 \mathrm{MHz}, \mathrm{CDCl}_{3}\right): \delta 1.01(\mathrm{~s}, 6 \mathrm{H}), 1.23$ $(\mathrm{s}, 6 \mathrm{H}), 1.98(\mathrm{~m}, 8 \mathrm{H}), 4.56(\mathrm{~s}, 1 \mathrm{H}), 7.52-7.98(\mathrm{~m}, 4 \mathrm{H})$, and 12.11 (s, ex. with $\left.\mathrm{D}_{2} \mathrm{O}, 2 \mathrm{H}\right) ;{ }^{13} \mathrm{C} \mathrm{NMR}\left(126 \mathrm{MHz}, \mathrm{CDCl}_{3}\right)$ : $\delta 25.23,26.45,30.31,31.98,47.01,114.79,120.90,122.22$, $128.99,132.93,134.94,145.23,148.37,189.73(\mathrm{C}=\mathrm{O}), 198.8$
$(\mathrm{C}=\mathrm{O})$; (EI) found: $\mathrm{M}^{+}, 413.1805, \mathrm{C}_{23} \mathrm{H}_{27} \mathrm{NO}_{6}$ requires $\mathrm{M}^{+}$, 413.1618; LRMS m/z (EI): $413\left(\mathrm{M}^{+}, 66 \%\right), 336\left(\mathrm{M}-\mathrm{C}_{6} \mathrm{H}_{5}\right.$, $55 \%)$. Elemental analysis: found (\%): C, 66.91; H, 6.86; N, 3.41. Calcd. for $\mathrm{C}_{23} \mathrm{H}_{27} \mathrm{NO}_{6}$ : C, 66.81; $\mathrm{H}, 6.58 ; \mathrm{N}, 3.39$.

2.6. 2, 2' -((2,4-Dinitrophenyl)methylene)bis(3-hydroxy-5,5dimethylcyclohex-2-enone) (3e). FT-IR (KBr) $v_{\max } 3453$, $3114,2925,1701$ (C=O), 1565, 1456, 1332, 1245, 915, 826, and $808 \mathrm{~cm}^{-1} ;{ }^{1} \mathrm{H}$ NMR $\left(500 \mathrm{MHz}, \mathrm{CDCl}_{3}\right): \delta 1.05$ (s, 6H), 1.31 $(\mathrm{s}, 6 \mathrm{H}), 2.24(\mathrm{~m}, 8 \mathrm{H}), 4.56(\mathrm{~s}, 1 \mathrm{H}), 7.65-8.20(\mathrm{~m}, 4 \mathrm{H})$, and 12.43 (s, ex. with $\left.\mathrm{D}_{2} \mathrm{O}, 2 \mathrm{H}\right) ;{ }^{13} \mathrm{C} \mathrm{NMR}\left(126 \mathrm{MHz}, \mathrm{CDCl}_{3}\right)$ : $\delta$ 26.30, 28.66, 30.40, 32.91, 45.01, 108.65, 114.65, 124.02, 128.56, 131.53, 132.74, 142.33, 144.37, 187.98 (C=O), 192.76 $(\mathrm{C}=\mathrm{O})$; (EI) found: $\mathrm{M}^{+}, 458.1805, \mathrm{C}_{23} \mathrm{H}_{26} \mathrm{~N}_{2} \mathrm{O}_{8}$ requires $\mathrm{M}^{+}$, 458.1709; LRMS m/z (EI): $458\left(\mathrm{M}^{+}, 75 \%\right), 386\left(\mathrm{M}-\mathrm{C}_{6} \mathrm{H}_{5}\right.$, $65 \%)$. Elemental analysis: found (\%): $\mathrm{C}, 60.34 ; \mathrm{H}, 5.83 ; \mathrm{N}$, 6.21. Calcd. for $\mathrm{C}_{23} \mathrm{H}_{26} \mathrm{~N}_{2} \mathrm{O}_{8}$ : C, 60.26; $\mathrm{H}, 5.72 ; \mathrm{N}, 6.11$.

2.7. 2, 2'-((4-Fluorophenyl)methylene)bis(3-hydroxy-5,5-dimethylcyclohex-2-enone) (3f). FT-IR (KBr) $v_{\max } 3523,3115$, 2865, $1706(\mathrm{C}=\mathrm{O}), 1535,1433,1298,1222,950,838$ and $816 \mathrm{~cm}^{-1} ;{ }^{1} \mathrm{H}$ NMR $\left(500 \mathrm{MHz}, \mathrm{CDCl}_{3}\right): \delta 1.02(\mathrm{~s}, 6 \mathrm{H}), 1.23$ (s, 6H), $2.42(\mathrm{~m}, 8 \mathrm{H}), 4.86(\mathrm{~s}, 1 \mathrm{H}), 7.02(\mathrm{~d}, \mathrm{~J}=8.01 \mathrm{~Hz}, 2 \mathrm{H})$, $7.24(\mathrm{~d}, \mathrm{~J}=8.01 \mathrm{~Hz}, 2 \mathrm{H})$, and 11.98 (s, ex. with $\left.\mathrm{D}_{2} \mathrm{O}, 2 \mathrm{H}\right) ;{ }^{13} \mathrm{C}$ $\mathrm{NMR}\left(126 \mathrm{MHz}, \mathrm{CDCl}_{3}\right.$ ): $\delta$ 27.32, 29.67, 31.41, 32.92, 47.01, $56.87,112.65,138.93,141.94,146.33,156254$ (d, J = 285.5 Hz, CF), 188.27 (C=O), $191.88(\mathrm{C}=\mathrm{O})$; (EI) Found: $\mathrm{M}^{+}, 386.1911$, $\mathrm{C}_{23} \mathrm{H}_{27} \mathrm{FO}_{4}$ requires $\mathrm{M}^{+}$, 386.1904; LRMS m/z (EI): $386\left(\mathrm{M}^{+}\right.$, $76 \%), 309\left(\mathrm{M}-\mathrm{C}_{6} \mathrm{H}_{5}, 45 \%\right)$. Elemental analysis: found (\%): C, 71.56; H, 7.12. Calcd. for $\mathrm{C}_{23} \mathrm{H}_{27} \mathrm{FO}_{4}$ : C, 71.48; H, 7.04.

2.8. 2,2'-((4-(Trifluoromethyl)phenyl)methylene)bis(3-hydrox$y$-5,5-dimethylcyclohex-2-enone) (3g). FT-IR (KBr) $v_{\max }$ 3556, 3121, 2865, 1698 (C=O), 1550, 1420, 1376, 1244, 927, 865 , and $831 \mathrm{~cm}^{-1} ;{ }^{1} \mathrm{H}$ NMR $\left(500 \mathrm{MHz}, \mathrm{CDCl}_{3}\right): \delta 1.12(\mathrm{~s}$, $6 \mathrm{H}), 1.29$ (s, 6H), $2.28(\mathrm{~m}, 8 \mathrm{H}), 4.76(\mathrm{~s}, 1 \mathrm{H}), 7.41-7.56(\mathrm{~m}$, $4 \mathrm{H})$, and 12.37 (s, ex. with $\left.\mathrm{D}_{2} \mathrm{O}, 2 \mathrm{H}\right) ;{ }^{13} \mathrm{C}$ NMR $(126 \mathrm{MHz}$, $\left.\mathrm{CDCl}_{3}\right): \delta 27.39,29.59,31.40,32.38,46.31,47.01,115.31$, $123.54,126.11\left(\mathrm{q}, \mathrm{J}=283.4 \mathrm{~Hz}, \mathrm{CF}_{3}\right), 131.55,136.68,148.98$, 184.57(C=O), $190.88(\mathrm{C}=\mathrm{O})$; (EI) found: $\mathrm{M}^{+}, 436.1905$, $\mathrm{C}_{24} \mathrm{H}_{27} \mathrm{~F}_{3} \mathrm{O}_{4}$ requires $\mathrm{M}^{+}$, 436.1910; LRMS m/z (EI): 436 $\left(\mathrm{M}^{+}, 86 \%\right), 359\left(\mathrm{M}-\mathrm{C}_{6} \mathrm{H}_{5}, 35 \%\right)$. Elemental analysis: found (\%): C, 66.01; $\mathrm{H}, 6.26$. Calcd. for $\mathrm{C}_{24} \mathrm{H}_{27} \mathrm{~F}_{3} \mathrm{O}_{4}$ : C, 66.04; $\mathrm{H}$, 6.24 .

2.9. 2, 2'-((4-Methoxyphenyl)methylene)bis(3-hydroxy-5,5dimethylcyclohex-2-enone) (3h). FT-IR (KBr) $v_{\max } 3421$, 3098, 2876, 1717 (C=O), 1552, 1450, 1362, 1291, 951, 835, and $823 \mathrm{~cm}^{-1} ;{ }^{1} \mathrm{H}$ NMR $\left(500 \mathrm{MHz}, \mathrm{CDCl}_{3}\right): \delta 1.15(\mathrm{~s}, 6 \mathrm{H})$, $1.51(\mathrm{~s}, 6 \mathrm{H}), 2.54(\mathrm{~m}, 8 \mathrm{H}), 4.78(\mathrm{~s}, 1 \mathrm{H}), 7.41-7.56(\mathrm{~m}$, $4 \mathrm{H})$, and 11.76 (s, ex. with $\left.\mathrm{D}_{2} \mathrm{O}, 2 \mathrm{H}\right) ;{ }^{13} \mathrm{C}$ NMR $(126 \mathrm{MHz}$, $\left.\mathrm{CDCl}_{3}\right): \delta 28.43,30.67,32.61,33.93,49.02,116.65,122.91$, $122.22,128.99,132.93,134.94,145.23,148.37,181.37$ $(\mathrm{C}=\mathrm{O}), 189.78(\mathrm{C}=\mathrm{O})$; (EI) found: $\mathrm{M}^{+}, 398.2111, \mathrm{C}_{24} \mathrm{H}_{30} \mathrm{O}_{5}$ requires $\mathrm{M}^{+}, 398.2103$; LRMS m/z (EI): $398\left(\mathrm{M}^{+}, 46 \%\right), 321$ $\left(\mathrm{M}-\mathrm{C}_{6} \mathrm{H}_{5}, 55 \%\right)$. Elemental analysis: found (\%): C, 72.43; $\mathrm{H}$, 7.64. Calcd. for $\mathrm{C}_{24} \mathrm{H}_{30} \mathrm{O}_{5}$ : C, 72.34; $\mathrm{H}, 7.59$. 
<smiles>[X]c1ccc(C=O)cc1</smiles>

$\mathrm{X}=\mathrm{H}, 4-\mathrm{Cl}, 3-\mathrm{NO}_{2}, 4-\mathrm{NO}_{2}, 2,4-\left(\mathrm{NO}_{2}\right)_{2}, 4-\mathrm{F}, 4-\mathrm{CF}_{3}, 4-\mathrm{OCH}_{3}, 4-\mathrm{CH}_{3}, 4-\mathrm{Br}$

Scheme 1: The Knoevenagel condensation between substituted benzaldehydes (1a-j) and dimedone (2) in presence of $\mathrm{ZrOCl}_{2}$. $8 \mathrm{H}_{2} \mathrm{O} / \mathrm{NaNH}_{2}$ as catalysis to yield the product $(3 \mathbf{a}-\mathbf{j})$.

2.10. 2, 2' -(p-Tolylmethylene)bis(3-hydroxy-5,5-dimethylcyclohex-2-enone) (3i). FT-IR (KBr) $v_{\max } 3427,3111,2901$, $1707(\mathrm{C}=\mathrm{O}), 1495,1450,1345,1264,943,835$ and $819 \mathrm{~cm}^{-1}$; ${ }^{1} \mathrm{H}$ NMR $\left(500 \mathrm{MHz}, \mathrm{CDCl}_{3}\right): \delta 1.04(\mathrm{~s}, 6 \mathrm{H}), 1.21(\mathrm{~s}, 6 \mathrm{H})$, $2.21(\mathrm{~m}, 8 \mathrm{H}), 4.51(\mathrm{~s}, 1 \mathrm{H}), 7.32-7.546(\mathrm{~m}, 4 \mathrm{H})$, and $11.12(\mathrm{~s}$, ex. with $\left.\mathrm{D}_{2} \mathrm{O}, 2 \mathrm{H}\right) ;{ }^{13} \mathrm{C}$ NMR $\left(126 \mathrm{MHz}, \mathrm{CDCl}_{3}\right): \delta 26.31$, $26.67,30.31,31.9,46.11,112.79,119.90,121.62,124.99$, $131.73,134.94,141.23,148.37,182.07(\mathrm{C}=\mathrm{O}), 189.08(\mathrm{C}=\mathrm{O})$; (EI) found: $\mathrm{M}^{+}, 382.4970, \mathrm{C}_{24} \mathrm{H}_{30} \mathrm{O}_{4}$ requires $\mathrm{M}^{+}, 382.4906$; LRMS $\mathrm{m} / \mathrm{z}(\mathrm{EI}): 382\left(\mathrm{M}^{+}, 43 \%\right), 305\left(\mathrm{M}-\mathrm{C}_{6} \mathrm{H}_{5}, 45 \%\right)$. Elemental analysis: found (\%): $\mathrm{C}, 75.64 ; \mathrm{H}, 8.04$. Calcd. for $\mathrm{C}_{24} \mathrm{H}_{30} \mathrm{O}_{4}$ : C, 75.36; $\mathrm{H}, 7.91$.

2.11. 2, $2^{\prime}$-((4-Bromophenyl)methylene)bis(3-hydroxy-5,5dimethylcyclohex-2-enone) (3j). FT-IR ( $\mathrm{KBr}) v_{\max } 3453$, 3121, 2908, 1710 (C=O), 1567, 1444, 1376, 1254, 917, 835, and $821 \mathrm{~cm}^{-1} ;{ }^{1} \mathrm{H}$ NMR $\left(500 \mathrm{MHz}, \mathrm{CDCl}_{3}\right): \delta 1.13(\mathrm{~s}, 6 \mathrm{H})$, $1.53(\mathrm{~s}, 6 \mathrm{H}), 2.62(\mathrm{~m}, 8 \mathrm{H}), 476(\mathrm{~s}, 1 \mathrm{H}), 7.65-7.87(\mathrm{~m}, 4 \mathrm{H})$, and 11.75 (s, ex. with $\left.\mathrm{D}_{2} \mathrm{O}, 2 \mathrm{H}\right) ;{ }^{13} \mathrm{C}$ NMR $\left(126 \mathrm{MHz}, \mathrm{CDCl}_{3}\right)$ : $\delta 24.33,28.76,31.31,32.9,47.04,115.81,121.91,122.02$, $128.99,132.93,134.94,145.23,148.37,187.79(\mathrm{C}=\mathrm{O}), 190.69$ $(\mathrm{C}=\mathrm{O})$; (EI) found: $\mathrm{M}^{+}, 446.1201, \mathrm{C}_{23} \mathrm{H}_{27} \mathrm{BrO}_{4}$ requires $\mathrm{M}^{+}$, 446.1128; LRMS m/z (EI): $446\left(\mathrm{M}^{+}, 56 \%\right), 336\left(\mathrm{M}-\mathrm{C}_{6} \mathrm{H}_{5}\right.$, $45 \%)$. Elemental analysis: found (\%): C, $61.82 ; \mathrm{H}, 5.96 ; \mathrm{Br}$, 17.474. Calcd. for $\mathrm{C}_{23} \mathrm{H}_{27} \mathrm{BrO}_{4}$ : C, 61.75; H, 6.08; $\mathrm{Br}, 17.86$.

\section{Results and Discussion}

In our continued interest, in the Knoevenagel condensations and its application in the synthesis of bioactive molecules, we report here a very simple and highly efficient method for the condensation of various aromatic (1) with active methylene compound (2), for example, dimedone, in the presence of $\mathrm{ZrOCl} / \mathrm{NaNH}_{2}$ as catalysis in THF at room temperature with stirring (Scheme 1).

It was exciting to observe that all the reactions occurred rapidly and were complete in just a few minutes giving excellent yields of the Knoevenagel products (3) (Table 1).

To determine the appropriate concentration of the catalyst zirconium oxychloride/sodium amide, we investigated the model reaction at different concentration of zirconium oxychloride/sodium amide such as 5, 10, 15, and $20 \mathrm{~mol} \%$.
TABLE 1: Reaction time, yields, and melting points of the products $(3 \mathbf{a}-\mathbf{j})$.

\begin{tabular}{lcccc}
\hline Entry & $\mathrm{X}$ & Time $(\min )$ & Yield $(\%)^{\mathrm{a}}$ & $\mathrm{Mp}\left({ }^{\circ} \mathrm{C}\right)$ \\
\hline 1 & $\mathrm{H}$ & 20 & 88 & $188-189$ \\
2 & $4-\mathrm{Cl}$ & 25 & 83 & $144-145$ \\
3 & $3-\mathrm{NO}_{2}$ & 20 & 90 & $151-152$ \\
4 & $4-\mathrm{NO}_{2}$ & 10 & 96 & $162-163$ \\
5 & $2,4-\left(\mathrm{NO}_{2}\right)_{2}$ & 5 & 97 & $172-173$ \\
6 & $4-\mathrm{F}$ & 15 & 90 & $183-184$ \\
7 & $4-\mathrm{CF}_{3}$ & 10 & 98 & $133-134$ \\
8 & $4-\mathrm{OCH}_{3}$ & 25 & 90 & $166-167$ \\
9 & $4-\mathrm{CH}_{3}$ & 30 & 91 & $178-179$ \\
10 & $4-\mathrm{Br}$ & 30 & 91 & $184-185$ \\
\hline
\end{tabular}

Reaction conditions: 1 (1 mmol), $2(1 \mathrm{mmol}), \mathrm{ZrOCl}_{2} \cdot 8 \mathrm{H}_{2} \mathrm{O} / \mathrm{NaNH}_{2}$ $(15 \mathrm{~mol} \%)$, in THF $(5 \mathrm{~mL})$ at $25^{\circ} \mathrm{C}$.

${ }^{\mathrm{a}}$ Isolated yield.

TABLE 2: Effect of concentrations of catalyst.

\begin{tabular}{lcc}
\hline Entry & $\mathrm{ZrOCl}_{2} \cdot 8 \mathrm{H}_{2} \mathrm{O}(\mathrm{mol} \%)$ & Yield $(\%)^{\mathrm{a}}$ \\
\hline 1 & 5 & 35 \\
2 & 10 & 65 \\
3 & 15 & 88 \\
4 & 20 & 88 \\
\hline
\end{tabular}

Reaction conditions: 1a $(1 \mathrm{mmol}), 2$ (1 mmol), $\mathrm{ZrOCl}_{2} \cdot 8 \mathrm{H}_{2} \mathrm{O} / \mathrm{NaNH}_{2}$ $(15 \mathrm{~mol} \%)$, in THF $(5 \mathrm{~mL})$ at $25^{\circ} \mathrm{C}$.

${ }^{\mathrm{a}}$ Isolated yields.

TABLE 3: Effect of temperature.

\begin{tabular}{lcc}
\hline Entry & Temperature $\left({ }^{\circ} \mathrm{C}\right)$ & Yield $(\%)^{\mathrm{a}}$ \\
\hline 1 & 0 & 35 \\
2 & 5 & 45 \\
3 & 10 & 48 \\
4 & 15 & 55 \\
5 & 20 & 60 \\
6 & 25 & 88 \\
7 & 35 & 75 \\
8 & 40 & 68 \\
\hline Reaction condition: 1a $(1 \mathrm{mmol}), \mathbf{2}(1 \mathrm{mmol}), \mathrm{ZrOCl}_{2} \cdot 8 \mathrm{H}_{2} \mathrm{O} / \mathrm{NaNH}_{2}$ \\
(15 mol \%), in THF $(5 \mathrm{~mL})$. &
\end{tabular}

The product was found in $35 \%, 65 \%, 88 \%$, and $88 \%$ yield, respectively. This indicates that $15 \mathrm{~mol} \%$ of zirconium oxychloride/sodium amide is sufficient to carry out the reaction smoothly (Table 2).

The temperature of $25^{\circ} \mathrm{C}$ was chosen as optimum temperature. Any further increase in the temperature failed to enhance the reaction rate substantially, while lowering the temperature below $25^{\circ} \mathrm{C}$ did show down the reaction rate (Table 3).

In order to evaluate the effect of solvent, various solvents such as dichloromethane, chloroform, ethyl acetate, methanol, water, and tetrahydrofurane were used for the 
TABLE 4: Screening of solvents.

\begin{tabular}{lccc}
\hline Entry & Solvent & Temperature $\left({ }^{\circ} \mathrm{C}\right)$ & Time $(\mathrm{h})$ \\
\hline 1 & Dichloromethane & 25 & Yield $(\%)^{\mathrm{a}}$ \\
2 & Chloroform & 25 & - \\
3 & Ethyl acetate & 25 & Trace \\
4 & Methanol & 25 & Trace \\
5 & Water & 25 & Trace \\
6 & Tetrahydrofurane (dry) & 25 & Trace \\
\hline
\end{tabular}

Reaction condition: $1 \mathrm{a}(1 \mathrm{mmol}), 2(1 \mathrm{mmol}), \mathrm{ZrOCl}_{2} \cdot 8 \mathrm{H}_{2} \mathrm{O} / \mathrm{NaNH}_{2}(15 \mathrm{~mol} \%)$, in solvent $(5 \mathrm{~mL})$ at $25^{\circ} \mathrm{C}$.

${ }^{\mathrm{a}}$ Isolated yields.

model reaction in the presence of zirconium oxychloride/sodium amide. Surprisingly, use of dichloromethane stopped the reaction at the Knoevenagel condensation step and no further reaction took place (TLC). Reaction in chloroform, ethyl acetate, methanol, and water resulted in moderate yields trace, whereas water brought the reaction to completion efficiently to furnish the product in excellent $88 \%$ yield (Table 4, entry 6).

\section{Conclusion}

In summary, we have developed an expedient and clean protocol for the synthesis of substituted $2,2^{\prime}$-(arylmethylene)bis(3-hydroxy-5,5-dimethylcyclohex-2-enone) derivatives. This method has the advantages of a wide scope of substrates, operational simplicity, easy work-up procedures, shorter reaction times, and high yields.

\section{Acknowledgment}

Generous support of this work by the Payame Noor University, Tehran, is gratefully acknowledged.

\section{References}

[1] B. M. Trost and I. Fleming, Comprehensive Organic Synthesis, vol. 1-9, Pergamon Press, New York, NY, USA, 1991.

[2] D. Basavaiah, A. J. Rao, and T. Satyanarayana, "Recent advances in the Baylis-Hillman reaction and applications," Chemical Reviews, vol. 103, no. 3, pp. 811-892, 2003.

[3] D. E. Sammelson and M. J. Kurth, "Carbon-carbon bondforming solid-phase reactions. Part II," Chemical Reviews, vol. 101, no. 1, pp. 137-202, 2001.

[4] R. R. Huddleston and M. J. Krische, "Enones as latent enolates in catalytic processes: catalytic cycloreduction, cycloaddition and cycloisomerization," Synlett, no. 1, pp. 12-21, 2003.

[5] S. E. . Gibson and A. Stevenazzi, "The Pauson-Khand reaction: the catalytic age is here!," Angewandte Chemie International Edition, vol. 42, no. 16, pp. 1800-1810, 2003.

[6] M. Moreno-Monas and R. Pleixats, "Formation of carboncarbon bonds under catalysis by transition-metal nanoparticles," Accounts of Chemical Research, vol. 36, no. 8, pp. 638-643, 2003.

[7] Z. Tang, Z.-H. Yang, X.-H. Chen et al., "A highly efficient organocatalyst for direct aldol reactions of ketones with aldedydes," Journal of the American Chemical Society, vol. 127, no. 25, pp. 9285-9289, 2005.
[8] A. M. Asiri, "Synthesis and characterization of dyes exemplified by 2-arylidene-1-dicyanomethyleneindane," Dyes and Pigments, vol. 42, no. 3, pp. 209-213, 1999.

[9] R. Ballini and G. J. Bosica, "Nitroaldol reaction in aqueous media: an important improvement of the henry reaction," The Journal of Organic Chemistry, vol. 62, no. 2, pp. 425-427, 1997.

[10] Y. Chi and S. H. Gellman, "Diphenylprolinol methyl ether: a highly enantioselective catalyst for michael addition of aldehydes to simple enones," Organic Letters, vol. 7, no. 19, pp. 4253-4256, 2005.

[11] H. Hattori, "Heterogeneous basic catalysis," Chemical Reviews, vol. 95, no. 3, pp. 537-558, 1995.

[12] F. Figueras, M. L. Kantam, and B. M. Choudary, "Solid base catalysts in organic synthesis," Current Organic Chemistry, vol. 10, no. 13, pp. 1627-1637, 2006.

[13] A. S. Canning, S. D. Jackson, E. McLeod, and E. M. Vass, "Aldol condensation of acetone over $\mathrm{CsOH} / \mathrm{SiO}_{2}$ : a mechanistic insight using isotopic labelling," Applied Catalysis A, vol. 289, no. 1, pp. 59-65, 2005.

[14] F. Winter, A. J. van Dillen, and K. P. de Jong, "Supported hydrotalcites as highly active solid base catalysts," Chemical Communications, no. 31, pp. 3977-3979, 2005.

[15] R. Garro, M. T. Navarro, J. Primo, and A. Corma, "Lewis acid-containing mesoporous molecular sieves as solid efficient catalysts for solvent-free Mukaiyama-type aldol condensation," Journal of Catalysis, vol. 233, no. 2, pp. 342-350, 2005.

[16] A. Smahi, A. Solhy, H. El Badaoui et al., "Potassium fluoride doped fluorapatite and hydroxyapatite as new catalysts in organic synthesis," Applied Catalysis A, vol. 250, no. 1, pp. 151-159, 2003.

[17] S. Wada and H. Suzuki, "Calcite and fluorite as catalyst for the Knövenagel condensation of malononitrile and methyl cyanoacetate under solvent-free conditions," Tetrahedron Letters, vol. 44, no. 2, pp. 399-401, 2003.

[18] M. L. Kantam, B. M. Choudary, C. V. Reddy, K. K. Rao, and F. Figueras, "Aldol and Knoevenagel condensations catalysed by modified Mg-Al hydrotalcite: a solid base as catalyst useful in synthetic organic chemistry," Chemical Communications, no. 9, pp. 1033-1034, 1998.

[19] K. Akutu, H. Kabashima, T. Seki, and H. Hattori, "Nitroaldol reaction over solid base catalysts," Applied Catalysis A, vol. 247, no. 1, pp. 65-74, 2003.

[20] B. M. Choudary, M. L. Kantam, C. V. Reddy, K. Koteswara Rao, and F. Figueras, "Henry reactions catalysed by modified $\mathrm{Mg}$-Al hydrotalcite: an efficient reusable solid base for selective synthesis of $\beta$-nitroalkanols," Green Chemistry, vol. 1, no. 4, pp. 187-189, 1999. 
[21] K. A. Utting and D. J. Macquarrie, "Silica-supported imines as mild, efficient base catalysts," New Journal of Chemistry, vol. 24, no. 8, pp. 591-595, 2000.

[22] S. M. Lai, C. P. Ng, R. Martin-Arnade, and K. L. Yeung, "Knoevenagel condensation reaction in zeolite membrane microreactor," Microporous and Mesoporous Materials, vol. 66, no. 2-3, pp. 239-252, 2003.

[23] T. Narender and K. P. Reddy, "A simple and highly efficient method for the synthesis of chalcones by using borontrifluoride-etherate," Tetrahedron Letters, vol. 48, no. 18, pp. 3177-3180, 2007.

[24] Y. Xia, Z.-Y. Yang, P. Xia, K. F. Bastow, Y. Nakanishi, and K.-H. Lee, "Antitumor agents. Part 202: novel $2^{\prime}$-amino chalcones: design, synthesis and biological evaluation," Bioorganic \& Medicinal Chemistry Letters, vol. 10, no. 8, pp. 699-701, 2000.

[25] F. Bios, C. Beney, A. Boumendjel, A. M. Marriotte, G. Conseil, and A. DiPietro, "Halogenated chalcones with high-affinity binding to P-glycoprotein: potential modulators of multidrug resistance," Journal of Medicinal Chemistry, vol. 41, no. 21, pp. 4161-4164, 1998.

[26] H.-K Hsieh, L.-T Tsao, and J.-P Wang, "Synthesis and antiinflammatory effect of chalcones," Journal of Pharmacy and Pharmacology, vol. 52, no. 2, pp. 163-171, 2000.

[27] F. Herencia, M. L. Ferrandiz, A. Ubeda et al., "Synthesis and anti-inflammatory activity of chalcone derivatives," Bioorganic \& Medicinal Chemistry Letters, vol. 8, no. 10, pp. 1169-1174, 1998.

[28] S. Ducki, R. Forrest, J. A. Hadfield et al., "Potent antimitotic and cell growth inhibitory properties of substituted chalcones," Bioorganic \& Medicinal Chemistry Letters, vol. 8, no. 9, pp. 1051-1056, 1998.

[29] L.-M Line, Y. Zhou, M. T. Flavin, L.-M Zhou, W. Nie, and F.-C Chen, "Chalcones and flavonoids as anti-tuberculosis agents," Bioorganic \& Medicinal Chemistry, vol. 10, no. 8, pp. 2795-2802, 2002.

[30] C. Furman, J. Lebeau, J.-C. Fruchart et al., "Di-tertbutylhydroxylated flavonoids protect endothelial cells against oxidized LDL-induced cytotoxicity," Journal of Biochemical and Molecular Toxicology, vol. 15, no. 5, pp. 270-278, 2001.

[31] E. J. Park, H. R. Park, J. S. Lee, and J. Kim, "Licochalcone A: an inducer of cell differentiation and cytotoxic agent from Pogostemon cablin," Planta Medica, vol. 64, no. 5, pp. 464-466, 1998.

[32] J. Rojas, M. Paya, J. N. Dominguez, and M. L. Ferrandiz, "The synthesis and effect of fluorinated chalcone derivatives on nitric oxide production," Bioorganic \& Medicinal Chemistry Letters, vol. 12, no. 15, pp. 1951-1954, 2002.

[33] M. Satyanarayana, P. Tiwari, B. K. Tripathi, A. K. Srivastava, and R. Pratap, "Synthesis and antihyperglycemic activity of chalcone based aryloxypropanolamines," Bioorganic \& Medicinal Chemistry, vol. 12, no. 5, pp. 883-889, 2004.

[34] S. F. Nielsen, S. B. Christensen, G. Cruciani, A. Kharazmi, and T. Liljefors, "Antileishmaniai chalcones: statistical design, synthesis, and three-dimensional quantitative structure-activity relationship analysis," Journal of Medicinal Chemistry, vol. 41, no. 24, pp. 4819-4832, 1998.

[35] X. Bu, L. Zhao, and Y. Li, "A facile synthesis of 6-C-prenylflavanones," Synthesis, no. 11, pp. 1246-1248, 1997.

[36] S. Sathyanarayana and H. G. Krishnamurthy, "Corroborative studies on the highly efficient preparation of 2' -hydroxychalcones using partially dehydrated barium hydroxide catalyst," Current Science, vol. 57, no. 20, pp. 1114-1116, 1988.
[37] A. R. Alcantara, J. M. Marinas, and J. V. Sinisterra, "Synthesis of $2^{\prime}$-hydroxychalcones and related compounds in interfacial solid-liquid conditions," Tetrahedron Letters, vol. 28, no. 14, pp. 1515-1518, 1987.

[38] M. J. Climent, A. Corma, S. Iborra, and J. Primo, "Base catalysis for fine chemicals production: claisen-schmidt condensation on zeolites and hydrotalcites for the production of chalcones and flavanones of pharmaceutical interest," Journal of Catalysis, vol. 151, no. 1, pp. 60-66, 1995.

[39] J. B. Daskiewicz, G. Comte, D. Barron, A. D. Pietro, and F. Thomasson, "Organolithium mediated synthesis of prenylchalcones as potential inhibitors of chemoresistance," Tetrahedron Letters, vol. 40, no. 39, pp. 7095-7098, 1999.

[40] A. Kossir and H. Qumimoum, "Dramatic activity enhancement of natural phosphate catalyst by lithium nitrate. An efficient synthesis of chalcones," Catalysis Communications, vol. 3, no. 8, pp. 335-339, 2002.

[41] N. O. Calloway and L. D. Green, "Reactions in the presence of petallic halides. I. $\beta$-unsaturated ketone formation as a side reaction in friedel-crafts acylations," Journal of the American Chemical Society, vol. 59, no. 5, pp. 809-811, 1937.

[42] T. Szell and I. Sohar, "New nitrochalcones. IX," Canadian Journal of Chemistry, vol. 47, no. 7, pp. 1254-1258, 1969.

[43] K. Irice and K. Watanabe, "Aldol condensations with metal(II) complex catalysts," Bulletin of the Chemical Society of Japan, vol. 53, no. 5, pp. 1366-1371, 1980.

[44] L. Mazza and A. Guaram, "An improved synthesis of 1,3diphenyl-2-buten-1-ones ( $\beta$-methylchalcones)," Synthesis, vol. 1980, no. 1, pp. 41-44, 1980.

[45] T. Nakano, S. Irifune, S. Umano, A. Inada, Y. Ishii, and M. Ogawa, "Cross-condensation reactions of cycloalkanones with aldehydes and primary alcohols under the influence of zirconocene complexes," Journal of Organic Chemistry, vol. 52, no. 11, pp. 2239-2244, 1987.

[46] N. Iranpoor and F. Kazemi, " $\mathrm{RuCl}_{3}$ catalyses aldol condensations of aldehydes and ketones," Tetrahedron, vol. 54, no. 32, pp. 9475-9480, 1998.

[47] M. R. Poor Heravi, "Selectfluor promoted synthesis of 9aryl-1,8-dioxooctahydroxanthane derivatives under solventfree conditions," Journal of the Iranian Chemical Society, vol. 6, no. 3, pp. 483-488, 2009. 

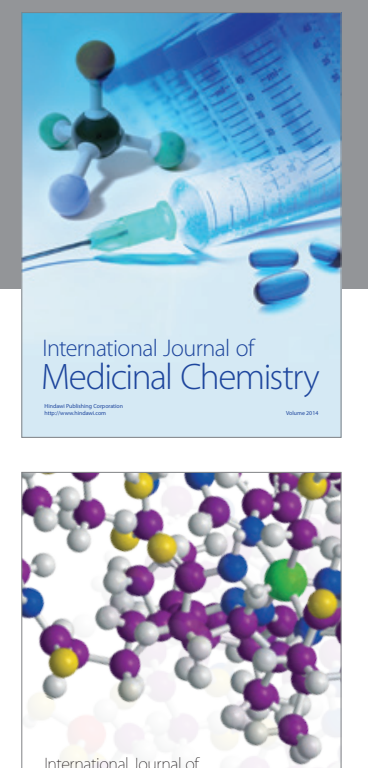

\section{Carbohydrate} Chemistry

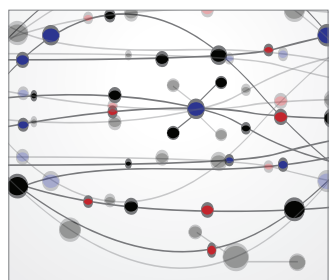

The Scientific World Journal
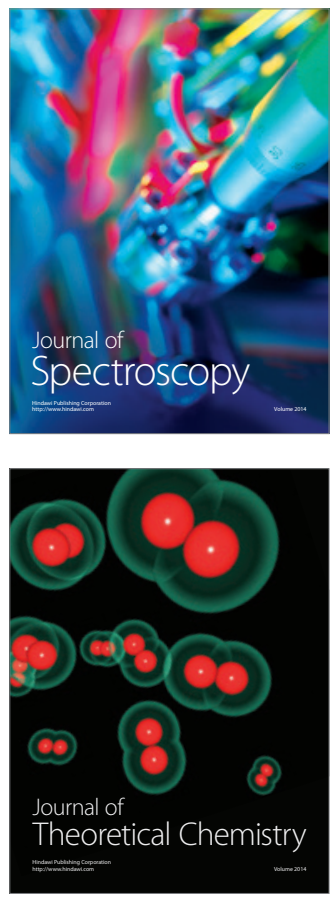
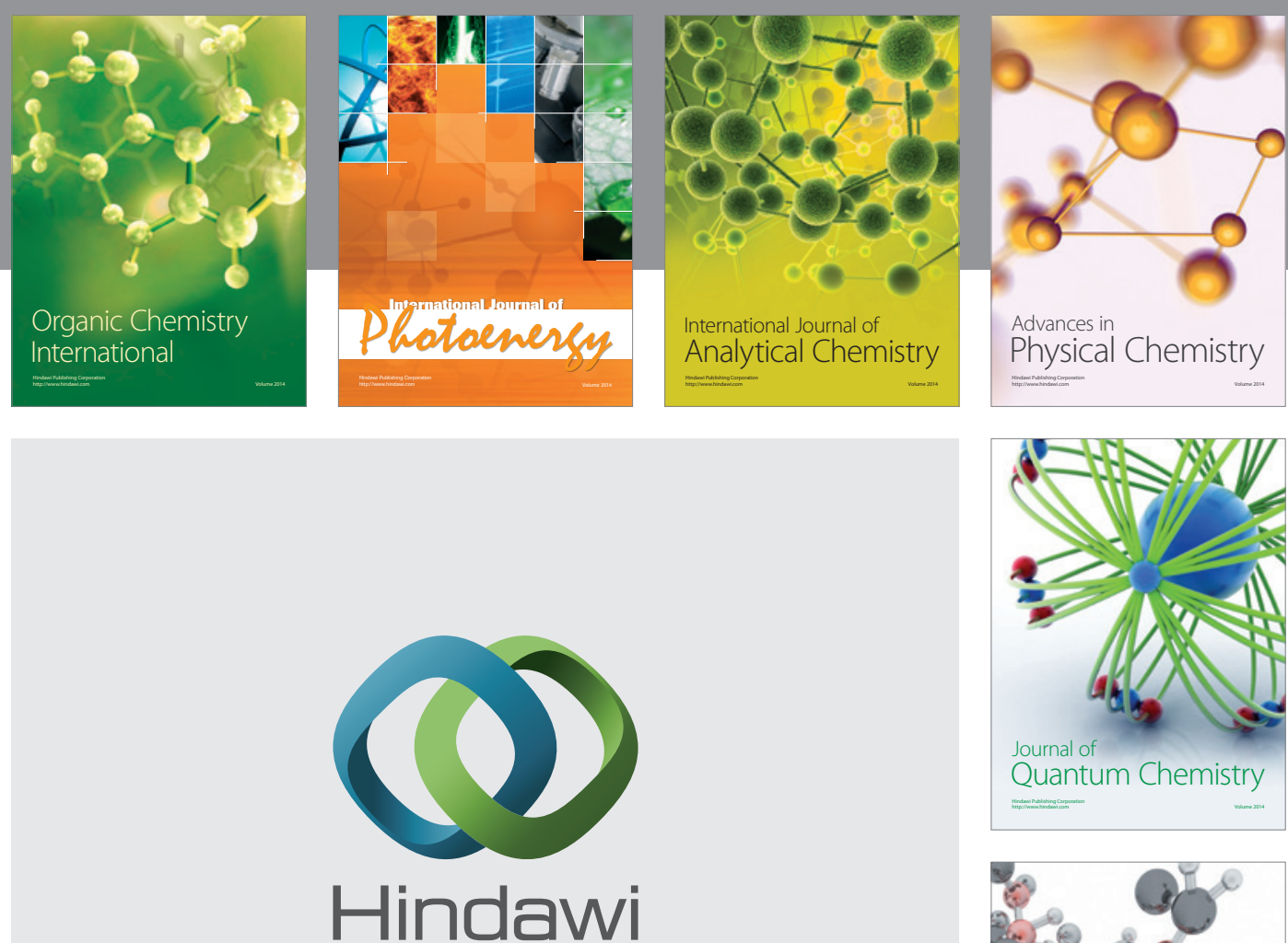

Submit your manuscripts at

http://www.hindawi.com

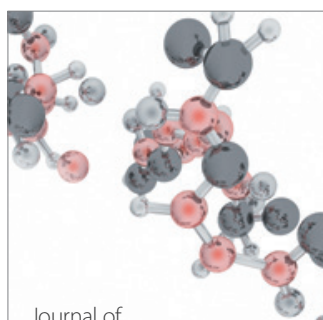

Analytical Methods

in Chemistry

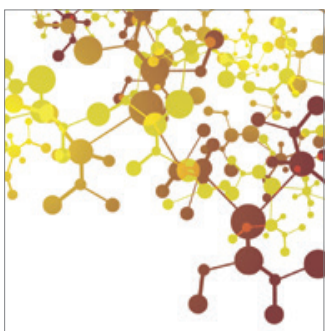

Journal of

Applied Chemistry

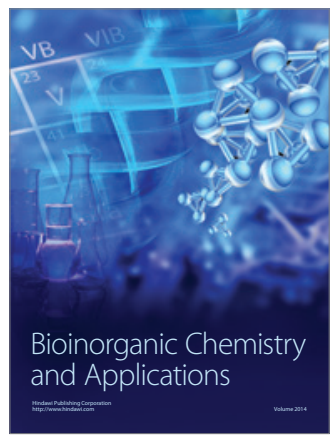

Inorganic Chemistry
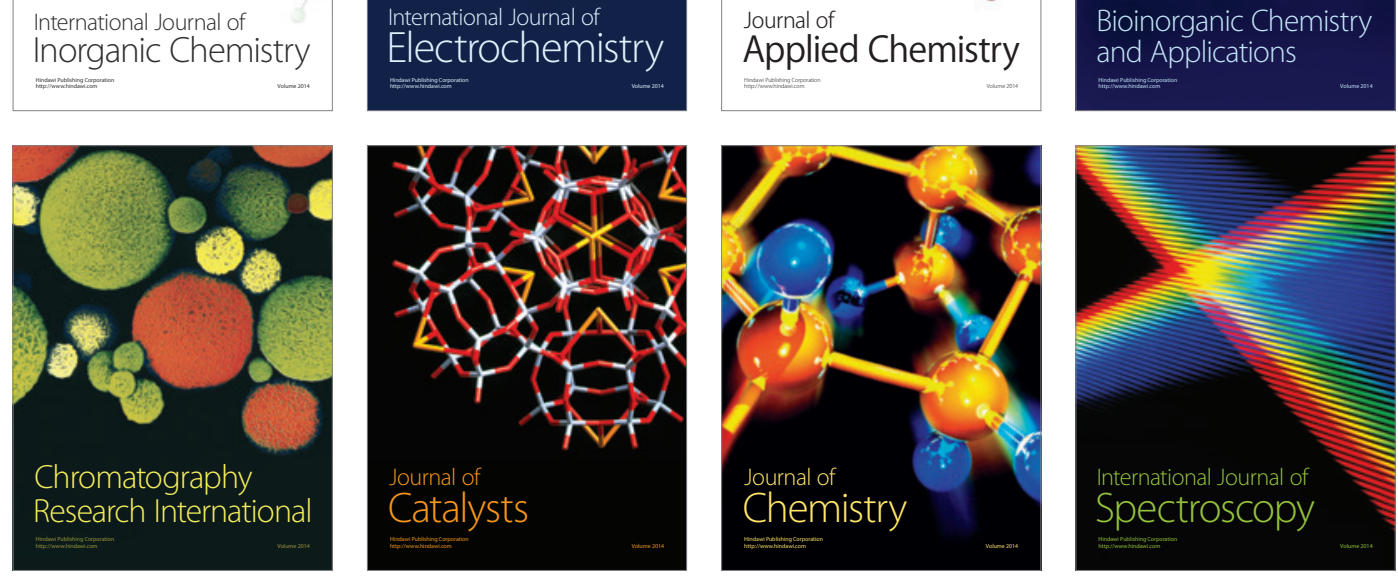